\title{
VARIA
}

\section{SOBRE LA PROCEDENCIA DE LOS PAISAJES DE JOSÉ DE RIBERA DE LA COLECCIÓN CASA DE ALBA Y OTRAS NOTICIAS}

\author{
Ma Del Mar Nicolás MartíneZ \\ Universidad de Almería \\ mnicolas@ual.es
}

\begin{abstract}
Se da a conocer, a través de una serie de documentos notariales, la procedencia en origen de los cuadros Paisajes con fortín y Paisajes con pastores, de José de Ribera, que formaron parte de la colección de pintura de don Fernando Joaquín Fajardo, sexto marqués de los Vélez y virrey de Nápoles.

Palabras claves: Pintura. Siglo XVII; José de Ribera; Marqués de los Vélez; Isabel Rosa de Ayala; María Teresa Pacheco; Ducado de Alba.
\end{abstract}

\section{ON THE PROVENANCE OF JOSÉ DE RIBERA'S LANDSCAPES IN THE COLLECTION OF THE HOUSE OF ALBA AND OTHER ISSUES}

The aim of this paper is to make known, through a series of notarial documents, the original provenance of José de Ribera's paintings Landscape with Fortress and Landscape with Shepherds, which formed part of the collection of paintings of Don Fernando Joaquín Fajardo, sixth marquis of Los Vélez and viceroy of Naples.

Key words: Painting; $17^{\text {th }}$ Century; José de Ribera; Marquis of Los Vélez; Isabel Rosa de Ayala; María Teresa Pacheco; Dukedom of Alba.

En el epígrafe Una silla para dos Riberas del discurso leído el 5 de marzo de 1984 por don Jesús Aguirre y Ortiz de Zárate (1934-2001), duque de Alba, en la recepción pública celebrada con motivo de su ingreso en la Real Academia de Bellas Artes de San Fernando', el nuevo académico dio a conocer a la opinión pública dos cuadros de José de Ribera (1591-1652), Paisaje con fortín (fig. 1) y Paisaje con pastores ${ }^{2}$ (fig. 2), que hasta entonces habían permanecido olvi-

\footnotetext{
${ }^{1}$ Aguirre, 1984.

2 Paisaje con fortín. Óleo sobre lienzo, 127 x 269 cm. Firmado: “Jusepe de Ribera/F.1639”. Paisaje con pastores (1639). Óleo sobre lienzo, 128 x $269 \mathrm{~cm}$.
}

Copyright: (C) 2016 CSIC. Este es un artículo de acceso abierto distribuido bajo los términos de una licencia de uso y distribución Creative Commons Attribution (CC-by) España 3.0. 


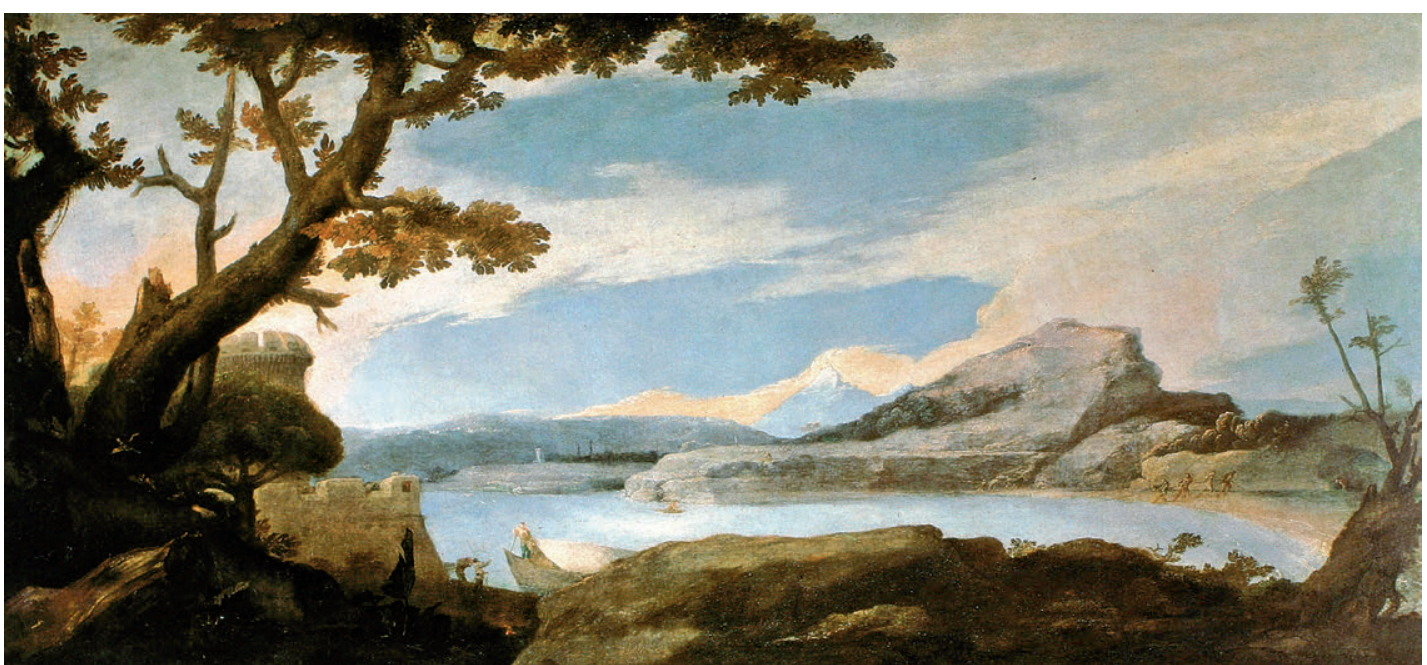

Fig. 1. José de Ribera, Paisaje con fortín, 1639. Fundación Casa de Alba.

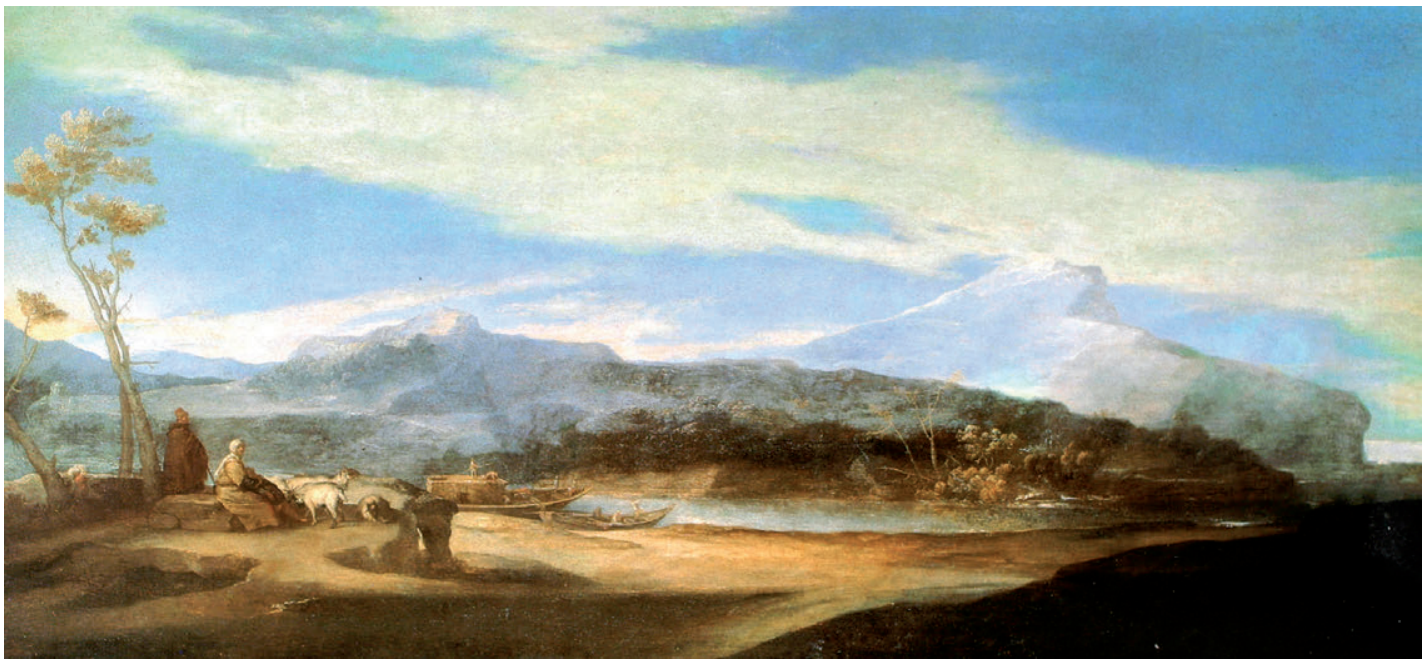

Fig. 2. José de Ribera, Paisaje con pastores, 1639. Fundación Casa de Alba.

dados, según sus palabras, en los pasillos del salmantino palacio de Monterrey, aunque se sabía de su existencia por don Jacobo Fitz-James Stuart (1878-1953), duque de Berwick y de Alba, al mencionarlos expresamente en su propio discurso de ingreso en la Real Academia de 25 de mayo de $1924^{3}$.

${ }^{3}$ Discursos leídos ante la Real Academia de Bellas Artes de San Fernando en la Recepción pública del Excmo. Sr. Duque de Berwick y de Alba, 25 de mayo de 1924, Archivo Real Academia de Bellas Artes de San Fernando (ARABBAASF), DIS 111-120: 87: "Países (Dos), uno con barca y el otro con cabaña y dos pastores (Al parecer, originales del Españoleto; 4 varas de ancho por 1 y $1 / 2$ de caída. Marcos dorados". 
La excelencia pictórica de tales obras, avalada unánimemente por los mejores especialistas en la producción artística del maestro español, y lo singular del tema, al ser las únicas pinturas conocidas de Ribera que tienen por motivo principal un paisaje, probablemente fantástico, en opinión de Nicola Spinosa, «pero plasmado con una concreción visual tal de los elementos naturales que parece como tomados de la realidad $»^{4}$, han hecho que estos cuadros sean de especial interés para los historiadores del arte, también por lo que concierne al asunto de su origen y procedencia. Sobre este último particular, la hipótesis mantenida hasta ahora por la historiografía artística ${ }^{5}$ había supuesto que los paisajes fueron encargados personalmente a José de Ribera por el VI conde de Monterrey ${ }^{6}$, pasando después a manos de su pariente, don Francisco de Zúñiga (†1662), conde de Miranda y demás títulos ${ }^{7}$. Sin embargo, esta presunción, reforzada por la circunstancia de que dichas pinturas aparecen inventariadas y tasadas el 16 de abril de 1755 por el pintor de cámara Andrés de la Calleja, entre las propias de doña María Teresa Pacheco (1706-1755), condesa de Miranda ${ }^{8}$, hay que rechazarla categóricamente a tenor de lo contenido en una serie de documentos recientemente

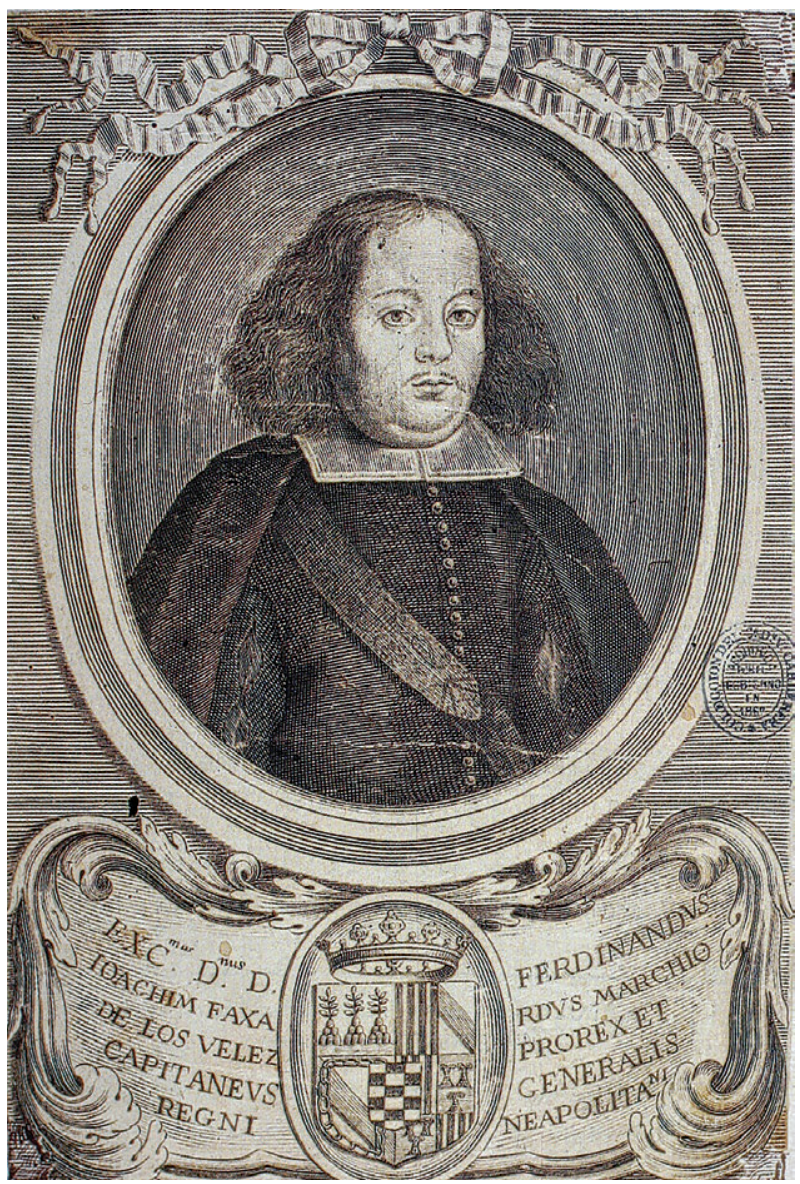

Fig. 3. Anónimo italiano, Fernando Joaquín Fajardo, VI marqués de los Vélez, c. 1675-1682, Madrid, Biblioteca Nacional. analizados, que informan sobre el primer propietario conocido de estas pinturas, el VI marqués de los Vélez, y de cómo ingresaron, junto con otras obras de su colección de arte, en el patrimonio de la Casa de Alba, lo que seguidamente se contextúa en este trabajo.

El 2 de noviembre de 1693 moría don Fernando Joaquín Fajardo (1635-1693), VI marqués de los Vélez y virrey de Nápoles entre 1675 y $1683^{9}$ (fig. 3). Después del óbito, sus testamentarios,

\footnotetext{
${ }^{4}$ Spinosa, 2008: 439 .

5 Aguirre, 1984: 42-43. Spinosa, 1992: 49; 2008: 439. Portús, 2012: 143, entre otros.

${ }^{6}$ Don Manuel de Fonseca y Zúñiga (†1653), sexto conde de Monterrey y virrey de Nápoles entre 1631 y 1637.

${ }^{7}$ Don Francisco de Zúñiga y Avellaneda fue tercer marqués de la Bañeza, séptimo conde de Miranda y tercer duque de Peñaranda.

${ }^{8}$ Testamentaria de María Teresa Pacheco Girón Toledo y Portugal, 1755, Archivo Histórico de Protocolos de la Comunidad de Madrid (AHPCM), Tomo 18.777, fol. 63r.

9 Don Fernando Joaquín Fajardo de Requesens y Zúñiga, sexto marqués de los Vélez, fue virrey y capitán general de Orán en la plaza de Mazalquivir (1666-1672), virrey de Cerdeña (1672-1675), virrey de Nápoles (1675-1683), caballerizo mayor de la reina María Luisa de Orleáns, gobernador y presidente del Consejo de Indias (1685-1687) y presidente de la Superintendencia de Hacienda.
} 
entre ellos, el duque de Montalto, casado con doña María Teresa Fajardo (c.1644/1645-1715), hermana y única heredera del marqués, al fallecer éste sin descendencia legítima que le sucediera ${ }^{10}$, ordenaron levantar inventario general de los bienes y hacienda del difunto, en cuyo apartado de pinturas $^{11}$ se registran un total de 506 piezas que fueron adquiridas principalmente por el virrey durante su gobierno en la corte partenopea, entre ellas, un lote de 42 obras de Luca Giordano, una tabla «pequeña» de la Resurrección del Señor atribuida a Miguel Ángel, un Descendimiento de la cruz de Andrea del Sarto o una tabla de Nuestra Señora de Giulio Romano, citadas aquí a modo de ejemplo ${ }^{12}$.

Aunque resulta arriesgado en el actual estado de la cuestión autentificar la correspondencia que se expresa en la relación, puesto que la colección histórica del marqués de los Vélez se dispersó tras su muerte, al liquidarse sus bienes por declaración de concurso de acreedores, hay que tener en cuenta la posibilidad de que los datos sean ciertos, pues, hasta ahora, todas las pinturas estudiadas, caso de San Genaro en gloria (1676) de Luca Giordano de la catedral de Valladolid ${ }^{13}$, o de Cain matando a Abel, original del pintor napolitano Francesco di Maria (c.1623-1690), recientemente localizada por Jesús Urrea en la iglesia mayor vallisoletana ${ }^{14}$, se ajustan fielmente a lo que se apunta en los asientos del inventario post-mortem del virrey, lo que también se puede afirmar de los dos cuadros de José de Ribera que nos ocupa, registrados, y por este orden, de la siguiente manera: «[una] pintura de un Pays de mano de Rivera de diez palmos y seis con marco dorado y negro tassada en 100 [ducados] [y] otra Pintura igual en todo tassada en 100 [ducados] $\rangle^{15}$.

Las medidas de dichas obras, que se corresponden bien con las propias de los lienzos de la colección Alba, teniendo en cuenta el margen de error -aceptado, por otra parte- de los antiguos inventarios, permitió en su momento identificar de manera inequívoca estos «payses» con los paisajes encontrados en el palacio de Monterrey ${ }^{1}$, unas pinturas que probablemente compró el marqués, como ya se ha dicho, durante su virreinato en Nápoles, ya que no parece que ingresaran en su patrimonio artístico por legado o testamentaría, como sí lo hicieron, por el contrario, otros cuadros del Españoleto de la antigua colección de sus padres, don Pedro Fajardo (1602-1647), V marqués de los Vélez y virrey de Sicilia y doña Mariana Engracia de Toledo y Portugal (†1686) ${ }^{17}$, aya del rey Carlos II, cuya imagen conocemos, junto con la de su hija María Teresa, por sendos retratos de La reina Mariana de Austria (1666) (fig. 4) y de La emperatriz Margarita de Austria (1665/6) pintados por Juan Bautista Martínez del Mazo ${ }^{18}$.

${ }^{10}$ María Teresa Fajardo Toledo y Portugal fue dama menina de la reina, séptima marquesa de los Vélez y octava duquesa de Montalto por su matrimonio, en 1664, con don Fernando de Aragón y Moncada.

${ }^{11}$ El inventario y tasación de las pinturas dio comienzo el 7 de diciembre de 1693 a cargo de los pintores Jorge Velarde y Francisco Pérez Sierra $(† 1709)$.

${ }^{12}$ Nicolás, 2012a: 155-189.

${ }^{13}$ Nicolás, 2012a: 170.

${ }^{14}$ Urrea, 2014: 60.

${ }^{15}$ Nicolás, 2012a: 174.

${ }^{16}$ Nicolás, 2012a:166-167.

${ }^{17}$ Don Fernando Joaquín Fajardo poseyó, al menos, un conjunto de 10 cuadros atribuidos a José de Ribera que fueron, además de los dos Paisajes que aquí se tratan, los siguientes: un San Jerónimo «de dos y medio y tres palmos», una Cabeza de San Juan Bautista «de dos y dos palmos y medio» ambos heredados de sus padres, un San Andrés de «dos y medio y tres palmos» y un San Francisco de Paula «compañera de la precedente», todo lo cual fue adjudicado en almoneda a don Diego Fernández de Villatoro, marqués del Castillo, y luego agregado, en 1697, por dicho señor a los bienes de mayorazgo establecido a favor de su sobrino, el conde de Pineda. También el marqués de los Vélez contó entre su pinturas con otras dos atribuidas a Ribera, un San José «de tres y cuatro palmos» una Santa Teresa «de cuatro y cinco palmos», que por sus dimensiones parecen obras distintas a otros dos cuadros del mismo asunto, propiedad de la V marquesa de los Vélez, luego en su colección. Ver: Pérez, 1992: 87. Nicolás, 2012a: 155-189. Nicolás; 2012b: 411-442. Nicolás: 2012c: 391-395.

${ }^{18}$ Llorente, 2006:14. 


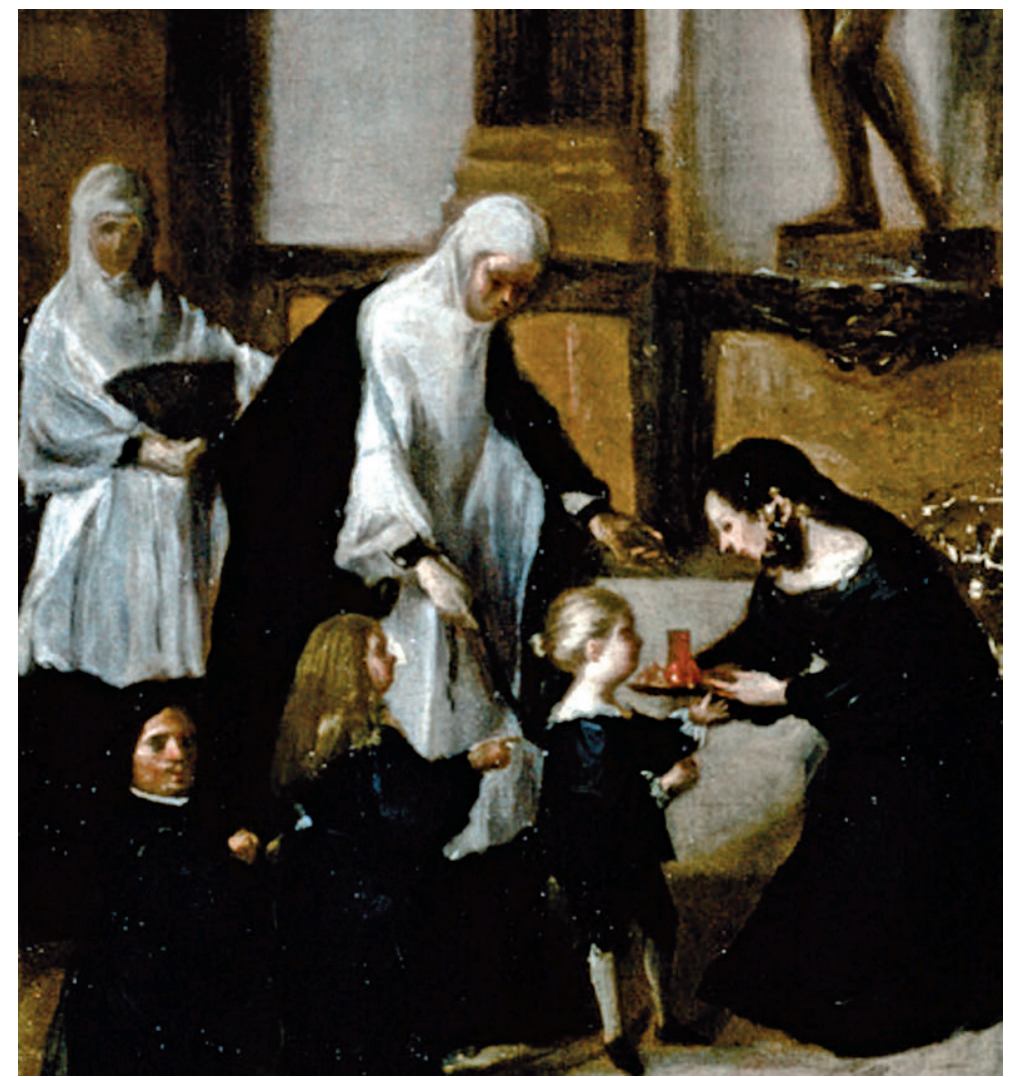

Fig. 4. Juan Bautista Martínez del Mazo, La reina Mariana de Austria (detalle), 1666, Londres, National Gallery.

Enlazando con lo dicho, el marqués de los Vélez contrajo segundas nupcias, el 3 de febrero de 1687, con doña Isabel Rosa de Ayala (c.1660-1717), dama de la reina Mariana de Habsburgo ${ }^{19}$. El incumplimiento de las capitulaciones matrimoniales por parte del novio, reconocido explícitamente en una de las clausulas de su testamento ${ }^{20}$, ocasionó que su viuda, casada de nuevo, desde el 6 de febrero de 1695, con don Joaquín José López de Zúñiga (1670-1725), marqués de la Bañeza ${ }^{21}$, entablara un pleito con los testamentarios del que fue su primer marido, a fin de cobrar la satisfacción de la dote y los gastos de cámara y alimentación que aún se le debía ${ }^{22}$, al tiempo que

${ }^{19}$ Licencia para contraer matrimonio entre Isabel Rosa de Ayala y el marqués de los Vélez. Patrimonio Nacional. Archivo General Palacio, Madrid (AGP), R(eal) C(apilla), caja 239, exp. 35. Véase, también: Sobre el matrimonio del marqués de los Vélez con la última hija de la condesa de Ayala, 1687, AGFCMS, Vélez, legajo 5582 y Expediente personal de Isabel Rosa de Ayala, (AGP), Per(sonal), caja 16.504, exp. 5. Isabel Rosa de Ayala fue hija del segundo matrimonio de don Fernando de Ayala y Toledo, tercer conde de Ayala y virrey de Sicilia (1660-1663), con doña Catalina Fajardo y Mendoza.

${ }^{20}$ Testamento que otorgó el Excelentísimo Señor marqués que fue de los Vélez Don Fernando Faxardo de Requesens, 2 de Noviembre de 1693, AHPCM, Tomo 13.703, fols.368r-382r. También: AGFCMS, Vélez, legajo 1272.

${ }^{21}$ Dos cartas del Conde de Miranda y Vizconde de la Calzada, Duque de Peñaranda, al Duque de Béjar, Juan Manuel López de Zúñiga, y a Pedro de Zúñiga, sus sobrinos, en la que participa el ajustado casamiento de su hijo el Marqués de la Bañeza con Isabel Rosa de Ayala. Madrid, 24 de enero de 1695, Archivo Histórico Nacional, Toledo (AHN), Sección Nobleza, Osuna, CT. 247, D.49.

${ }^{22}$ Pleito seguido por el conde de Miranda, duque de Peñaranda, como marido de doña Isabel Rosa de Ayala, sobre la paga y restitución de la dote, 1705, AGFCMS, Vélez, legajo 520. 


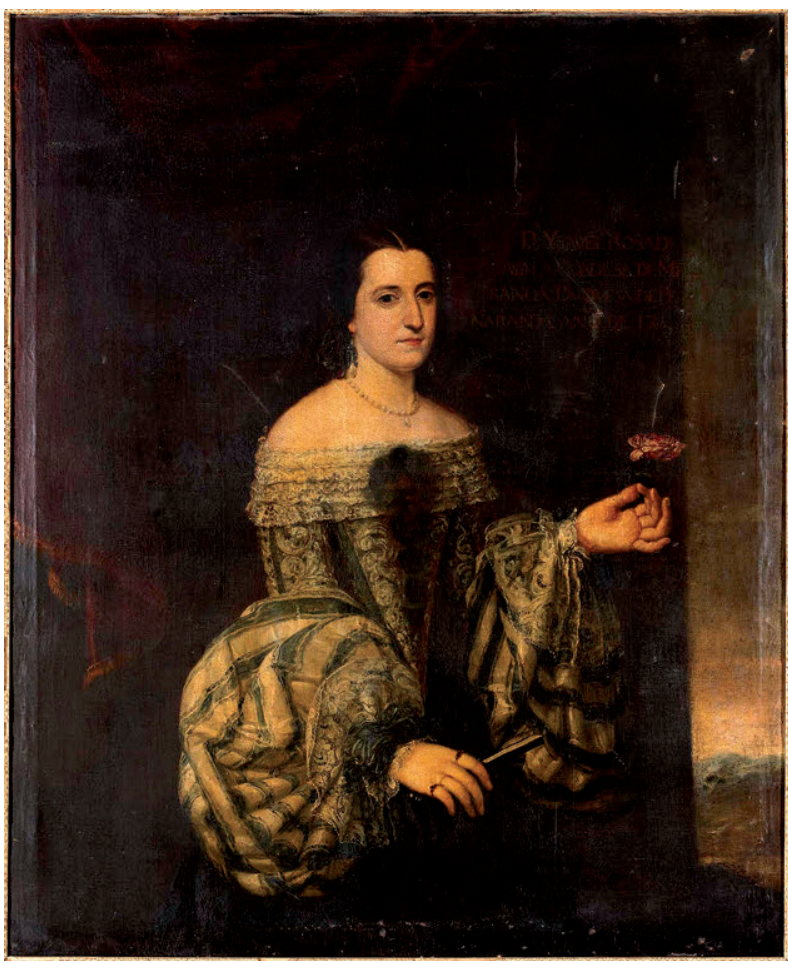

Fig. 5. Anónimo (escuela madrileña), Retrato de Isabel Rosa de Ayala, c. 1694-1700, Fundación Casa de Alba.

reclamó la entrega de parte de los legados que le había asignado el virrey en su testamento, de los que sólo tenía en su poder una joya formada por «un hilo de perlas que sirve de gargantilla con una mayor en forma de calabaza en medio y las dos arracadas correspondientes a dicho hilo que tiene dos perlas grandes en forma de calabaza» ${ }^{23}$.

Sobre este particular, la Casa de Alba posee una digna pintura de Isabel de Ayala retratada en pie, hasta la rodilla, vistiendo un conjunto formado por basquiña y jubón de ancho escote adornado de encaje, bajo el que sobresale unas amplísimas mangas rematadas en grandes puños de blonda por influencia de la moda francesa. La dama, de rasgos poco agraciados, aparenta una edad bien entrada en la treintena, va peinada con el cabello recogido en la nuca, sostiene en su mano izquierda una rosa alusiva a su segundo nombre de pila y se adorna con joyeles y con el magnífico aderezo de perlas y diamantes ante descrito (fig. 5). La obra, de escuela madrileña, presenta en el ángulo superior derecho la inscripción: «D. YSABEL ROSA DE/AYALA CONDESA DE MI/RANDA DUQUE-

SA DE PEÑA/NARANDA $1700 »^{24}$, que informa acerca de la nueva situación social de la retratada al recibir su marido, el marqués de la Bañeza, dichos títulos por la muerte de su madre, doña Ana María López de Zúñiga, en el año 1700. Esta data, que coincide con la que aparece en el letrero del cuadro, hace considerar que la pintura fuese ejecutada en fecha anterior a la que se presupone, tal vez, poco antes de la segunda boda de la marquesa de los Vélez, lo que justificaría desde el punto de vista de la representación que luzca joyas propias de la Casa de su primer marido, siendo factible que la leyenda fuese añadida posteriormente a título conmemorativo ${ }^{25}$.

El retrato de la condesa de Miranda -al igual que otras obras de arte que pertenecieron a la que fue marquesa de los Vélez- quedó incorporado por ciertas cuestiones legales al patrimonio de su hija política, la ya nombrada María Teresa Pacheco, como así consta en su inventario de bienes ${ }^{26}$. Casada en 1726 con don Antonio López de Zúñiga, conde de Miranda y duque de Peñaranda, tuvo

\footnotetext{
${ }^{23}$ Nicolás, 2012c: 388.

${ }^{24}$ Hermoso, 2009: 151.

${ }^{25}$ Agradezco a la Fundación Casa de Alba que nos haya remitido para su estudio y publicación la fotografía del Retrato de Isabel Rosa de Ayala.

${ }^{26}$ Testamentaría de María Teresa Pacheco Girón Toledo y Portugal, 1755, AHPCM, Tomo 18.777, fol. 66r-v.

${ }^{27}$ Posesiones dadas a la Exma. Sra. D ${ }^{a}$ María Manuela Kirkpatrick, condesa viuda de Montijo, como madre, tutora y cuidadora de la Exma. Sra. D a María Francisca de Sales Guzmán y Portocarrero, de los títulos, estados y Mayorazgos de las Casas de Montijo, Miranda... 1839, AHPCM, Tomo 24.757, fol. 455r.
} 
de su matrimonio cuatro vástagos, entre ellos, una hija llamada María Josefa, mujer que fue de don Cristóbal Portocarrero Guzmán, marqués de Valderrábanos y heredero del condado de Montijo. Después, extinguida la sucesión de las Casas de Miranda y Peñaranda por línea directa en la figura de doña María del Carmen Josefa de Zúñiga (†1829), siguió en todos los títulos don Cipriano de Palafox y Portocarrero (1784-1839), conde de Montijo, a quien sucedió y heredó su primogénita, María Francisca de Sales Guzmán y Portocarrero (1825-1860) ${ }^{27}$, duquesa de Alba por su matrimonio con don Jacobo Fitz-Stuart y Portocarrero $(1821-1881)^{28}$, razón por la cual ingresó en la colección de los duques de Berwick el conjunto de obras de arte que a continuación se refiere.

Se documenta que doña Isabel de Ayala obtuvo de los testamentarios de su primer marido la totalidad del legado establecido por el marqués de los Vélez a su favor, consistente en un grupo escultórico de «Nuestra Señora, San José y el Niño», una imagen de Nuestra Señora de la Asunción sobre trono de ángeles y un Ecce Homo, todo de escuela napolitana. También recibió, como primera compensación económica a sus demandas judiciales, una tapicería rica de La Historia de Elías «fina de Bruselas» y otra de La Historia del rey David, igualmente «fina de Bruselas, de dibujo de Rubens», ambas datadas con anterioridad a 1681, cuyo primer registro de propiedad se constata en el inventario post-mortem del virrey Fajardo de $1693^{29}$. Estos tapices figuran en la relación de bienes de María Teresa Pacheco -al igual que las esculturas antes citadas $-{ }^{30} \mathrm{y}$, al menos, hasta 1924, engrosaron la colección de los duques de Alba, aunque no hay constancia de su paradero actual, ni que hayan sido publicados ${ }^{31}$.

Pero por lo que fundamentalmente interesa, no sería hasta después de la muerte de la que fue marquesa de los Vélez y condesa de Miranda, cuando sus tres hijos recibieron las pinturas de los «dos Países de Marina de mano de Joseph de Rivera con sus marcos dorados y negro [tasados] en dos mil y doscientos reales de vellón» que, junto con otro lote de obras de la colección, a saber, una pintura de Federico Zuccaro, ocho dibujos de Polidoro de Caravaggio, un paisaje de la ciudad antigua de Nínive, el retrato del marqués de los Vélez original de Alberto Arnone, un Calvario italiano de bronce, un bajorrelieve de barro cocido con dieciséis putti jugando, dos Crucifijos de ámbar, una fuente de concha, varios relojes y una «uña de la gran bestia», les fueron entregadas a los demandantes por remate y liquidación de una deuda de 152.710 reales de vellón que aún se les debía sobre la satisfacción de la dote, arras y bienes gananciales de su madre, según consta en la escritura de transacción, ajuste y convenio, de fecha 20 de agosto de 1726 , firmada con doña

${ }^{28}$ Escritura de capitulaciones matrimoniales para el que han de contraer matrimonio el Excmo. Sr. D Jacobo Luis Stuart, Duque de Berwick y de Alba y la Excma. Sra. D ${ }^{a}$ Francisca de Sales Portocarrero y Palafox, Condesa de Montijo $y$ de Miranda, 1844, AHPCM, Tomo 25.268, fol. 151r.

${ }^{29}$ Inventario de tapicerías, alcatifas, alfombras, tapetes y reposteros, 1693, AGFCMS, Vélez, legajo 1657, pieza 6: «Otra Tapicería de ocho Annas de cayda de figuras grandes de la historia de Elías Profeta fina de Bruselas, que tiene una tarjeta en las cenefas de arriba con sus rótulos en los que dize el Misterio de cada paño; $Y$ en todos los dichos paños tiene cincuenta Anas de corrida que hacen en cuadro Trescientas Anas tasadas en 18.000 [reales] «. «Otra tapicería de ocho paños y seis anas de cayda fina de Bruselas de dibujo de Rubens de figuras grandes de la Historia de David con unas estatuas en las cenefas de la caída y tienen los dicho ocho paños cincuenta Anas y una Tercia de corrida poco más o menos por estar colgado, que hace en cuadro trescientas y una anas y dos tercias tasada en 15.495 [reales]». Puede consultarse: Nicolás, 2012a: 158-159.

${ }^{30}$ Testamentaria de María Teresa Pacheco Girón Toledo y Portugal, 1755, AHPCM, Tomo 18.777, fols, 169v-170r. El tasador de las tapicerías fue el maestro tapicero Francisco Pereda. Hay que advertir una discrepancia en los documentos consultados con relación al centro manufacturero donde fueron tejidos los tapices, que, en opinión de la doctora Concha Herrera, a la que agradezco sinceramente su opinión e inestimable ayuda en este punto del trabajo, debió de ser la ciudad de Amberes. Por su parte, el tasador de las esculturas fue el escultor de la corte Antonio Valeriano Moyano (†1770).

${ }^{31}$ Discursos leídos ante la Real Academia de Bellas Artes de San Fernando en la recepción pública del Excmo. Sr. Duque de Berwick y de Alba, 1924, ARABBAASF, DIS 111-120: 135. 
Catalina de Moncada y Aragón (1665-1728), VIII marquesa de Villafranca y de los Vélez ${ }^{32}$, cuya Memoria se transcribe íntegramente en el apéndice documental del presente trabajo.

\section{APÉNDICE DOCUMENTAL}

Archivo General Fundación Casa de Medina Sidonia. Vélez, legajo 4462

Cargos y datas de la testamentaría de don Fernando Joaquín Fajardo. 1722-1730.

MEMORIA

En 21 de febrero de 1726 el excelentísimo señor don Lope Núñez de Mendoza canónigo doctoral de Peñaranda con poder del Excelentísimo Señor don Antonio López de Zúñiga conde actual de Miranda y don Pedro de Ayuso y Rojas, presbítero, otorgaron ante Francisco Carrasco, escribano público en esta Corte, escriptura de transaczion, ajuste y convenio y el dicho don Pedro con poder de la Excelentísima Señora doña Catalina de Moncada y Aragón Marquesa de Villafranca y de los Vélez, duquesa de Montalto, en razón de las pretensiones debidas por Parte de dicho señor Conde de Miranda y sus hermanos contra los bienes, efectos y rentas de Concurso del Excelentísimo Señor Don Fernando Joachim Faxardo marqués que fue de los Vélez sobre la satisfacción de la Dote, Arras y Bienes gananciales de la Excelentísima Señora doña Isabel Rosa de Ayala, su Madre, Marquesa que fue de los Vélez y después condesa de Miranda, para lo que precedió una liquidación que está inserta en dicha escriptura. La que se aprobó por el Señor Don Francisco Bentura Esquivel y Arze del Consejo de S.M., su Alcalde de Casa y corte como Juez Particular y Privativo de este concurso en 29 de marzo de 1726 y se confirmó por los Señores del Real y Supremo Consejo de Castilla en 8 de Agosto de dicho año, y dicha liquidación resulta importar el cargo que se hizo por dicho Señor Conde de Miranda y sus hermanos al Concurso 1.361.732 reales y la data 1.060.004 Reales, y de Alcanze a favor de los Señores herederos de la Excelentísima Señora Doña Isabel Rossa de Ayala 301.728 reales de vellón los que por los motivos que en ellos se expresan se transfirieron en 152.710 reales vellón lo que reunieron en alhajas en la forma siguiente:

Primeramente una fuente de concha y ébano con cristales bronzeada y con figuras doradas demolido en noventa mil reales de vellón. Un Santísimo Cristo con su Santísima Madre, san Juan y la Magdalena todo de bronce dorado de Molido Cruz y Peana de ébano en quarenta y cuatro mil doscientos y veinte reales. Una pintura de mano de Sucaro con siete Ángeles tasada en doscientos ducados. Dos Payses de Marina de mano de Joseph de Rivera con sus marcos dorados y negros en dos mil y doscientos reales de vellón. Una perspectiva de la ciudad de Nínive con marco negro sin cristal en doscientos ducados. Un marco de peral con un Juego de Niños sin cristal en tres mil quinientos y cincuenta reales de vellón. Dos hechuras de Cruzifixos de ámbar con sus cajas en doscientos ducados. Una Uña de la Gran Bestia en cuarenta reales. Una bola de bronce dorada con un reloj en zeicientos reales de vellón. Un reloj de luz con flexo y reflexo con su Caja de ébano esmaltada con sus cañones y linternas en un mil y cien reales de vellón. Ocho dibujos Pinturas de noche romano de Polidoro en cuatro mil y cuatrocientos reales. Así mismo se entregó a don Lope Núñez de Mendoza el retrato del Señor Marqués de los Vélez.

Con los Ciento y Cinquenta y dos mill Setezientos y diez reales vellón de importe de las Alhajas arriba expresadas, se dieron por satisfechos el Señor Conde de Miranda y sus hermanos y otorgaron carta de Pago y finiquito a favor de esta testamentaría en Madrid, a 20 de agosto de 1726, ante Silvestre de Barrio, Escribano de Provincia y de esta comisión, la que está con los demás instrumentos ya zitados encuadernados y en la contaduría de esta Testamentaría.

\section{BIBLIOGRAFÍA}

Aguirre y Ortiz de Zárate, Jesús (1984): Discursos leidos ante la Real Academia de Bellas Artes de San Fernando en la recepción pública del Excelentísimo Señor don Jesús Aguirre y Ortiz de Zárate. Madrid: Academia de Bellas Artes de San Fernando.

Hermoso Romero, Ignacio (2009): "Perpetuar la memoria": retratos de la Casa de Alba . En: Marqués Ferrer, Virginia (coord.) (2009): Colección Casa de Alba. Sevilla: Junta de Andalucía, pp. 143-173.

32 Catalina de Moncada y Aragón fue hija única y heredera de Fernando de Aragón y Moncada y María Teresa Fajardo. Casó en 1680, en segundas nupcias, con don José Álvarez de Toledo, duque de Fernandina, después VIII marqués de Villafranca del Bierzo. Su nieto, José Álvarez de Toledo y Gonzaga, X marqués de Villafranca y de los Vélez, fue duque de Alba por su matrimonio con María del Pilar Teresa Cayetana de Silva, XIII duquesa de Alba. 
Llorente, Mercedes (2006): "Imagen y autoridad en una regencia. Los retratos de Mariana de Austria y los límites del poder". En: Studia histórica. Historia Moderna, 28, Salamanca, pp. 211-238.

Nicolás Martínez, Ma del Mar (2012a): "Las pinturas de la colección del VI Marqués de los Vélez. El inventario de bienes de 1693 y algunas otras consideraciones”. En: Camacho Martínez, Rosario / Asenjo Rubio, Eduardo (eds.) (2012): Patronos y modelos en las relaciones entre Andalucía, Roma y el Sur de Italia. Málaga: Ministerio de Economía y Competitividad, pp. 155-189.

Nicolás Martínez, Ma del Mar (2012b): “Los virreyes Fajardo y el coleccionismo artístico en Sicilia y Nápoles”. En: Camacho Martínez, Rosario / Asenjo Rubio, Eduardo / Calderón Roca, Belén (eds.): Fiestas y mecenazgo en las relaciones culturales del Mediterráneo en la Edad Moderna. Málaga: Ministerio de Economía y Competitividad, pp. 411-442.

Nicolás Martínez, Ma del Mar (2012c): "Los legados de arte y objetos suntuarios de Mariana Engracia de Toledo y María de Aragón, marquesas de los Vélez, y sus inventarios de bienes (1686)”. En: Rivas Carmona, Jesús (coord.): Estudios de Platería. Murcia: Universidad de Murcia, pp. 385-409.

Pérez Sánchez, Alfonso E. (1992): "Ribera y España". En: Pérez Sánchez, Alfonso E./Spinosa, Nicola (eds.): Ribera (1591-1652). Madrid: Museo del Prado, pp. 79-105.

Portús, Javier (2013): “Jusepe de Ribera (1591-1652). Paisaje con fortín. Paisaje con pastores". En: Calderón Ortega, José Manuel: El legado Casa de Alba. Madrid: Comunidad de Madrid, pp. 143-145.

Spinosa, Nicola (1992): "Ribera en Nápoles". En: Pérez Sánchez, Alfonso E. / Spinosa, Nicola (eds.): Ribera (15911652). Madrid: Museo del Prado, pp. 35-55.

Spinosa, Nicola (2008): Ribera. Obra completa. Madrid: Fundación Arte Hispánico.

Urrea, Jesús (2014): "Varia de arte y pasión en la catedral de Valladolid". En: Boletín de la Real Academia, 49, Valladolid, pp. 57-72.

Fecha de recepción: 03-VI-2015

Fecha de aceptación: 14-XII-2015 